\title{
ONDAS DE MONTAÑA EN LA CAPA LÍMITE PLANETARIA DE LA REGIÓN ANDINA VENEZOLANA.
}

\author{
HÉCTOR VÁSQUEZ ${ }^{1}$ E NELSON FALCÓN²
}

\author{
${ }^{1}$ Servicio de Meteorología de la Aviación Militar Bolivariana, Base Logística Aragua. Maracay, Edo. Aragua. \\ Venezuela \\ ${ }^{2}$ Laboratorio de Física de la Atmosfera y del Espacio Ultraterrestre, Dpto. de Física, Facultad Experimental \\ de Ciencias y Tecnología, Universidad de Carabobo, Valencia Edo. Carabobo, Venezuela.
}

\author{
nelsonfalconv@gmail.com
}

Recibido Abril de 2013 - Aceptado Octubre de 2014

\begin{abstract}
RESUMEN
Se evalúa las condiciones dinámicas y termodinámicas de la capa límite planetaria de la Región Andina Venezolana para la génesis de ondas de montaña. Para ello se calcula el número de Froude relacionando la estabilidad estática en presencia de accidentes orográficos con apoyo de perfiles verticales de viento, análisis de diagramas termodinámicos e imágenes de satélite. Se encuentra que cuando se presenta un flujo de aire perpendicular a la cordillera andina venezolana con vientos mayores a $10 \mathrm{~m} / \mathrm{s}$ bajo condiciones de estabilidad o inversión de temperatura sobre su cima, disponibilidad de humedad mayor a $70 \%$ desde superficie a su cumbre y numero de Froude ligeramente superior a 1, el resultado es la presencia de ondas de montaña asociadas a nubes lenticulares a sotavento de la cadena montañosa.
\end{abstract}

Palabras Claves: Ondas de Montaña; Numero de Froude; Andes Venezolanos.

\begin{abstract}
MOUNTAIN WAVES IN THE PLANETARY BOUNDARY LAYER OF THE VENEZUELAN ANDES.

Dynamic and thermodynamic conditions of the planetary boundary layer of the Venezuelan Andean region for the generation of mountain waves are evaluated. The Froude number is calculated by relating the static stability in the presence of orographic supported vertical wind profiles, diagrams and thermodynamic analysis of satellite images. It is found that when an air flow perpendicular to the Venezuelan Andes with winds greater than $10 \mathrm{~m} / \mathrm{s}$ under conditions of stability or temperature inversion over the top, availability of higher humidity to $70 \%$ from surface to its top and the number Froude slightly greater than 1 , the result is the presence of mountain waves associated with lenticular clouds at the leeward side of the mountain range.
\end{abstract}

Keywords: Waves Mountain; Froude number; Venezuelan Andes.

\section{INTRODUCCIÓN}

Las Ondas de montaña también conocidas como ondas de gravedad internas (OGI), ondas de sotavento o a veces ondas estacionarias de relieve; pueden ser definidas como un fenómeno ondulatorio en el flujo de aire perpendicularmente a una barrera montañosa (Gossard y Hooke, 1975), se forman al igual que las olas del mar, cuando una fuerza eleva la superficie de un fluido hasta un determinado nivel, a partir del cual la fuerza de gravedad lo hace descender produciéndose movimientos oscilatorios verticales, cuyas perturbaciones se propagan como ondas con longitudes variables entre 3 a $20 \mathrm{~km}$. Estas ondas son estables, manteniéndose dentro de ciertos límites su amplitud y longitud mientras se propagan corriente abajo. La ondulación de la corriente se hace débil a partir de $3 \mathrm{~km}$ por encima de la montaña, aunque en largas cadenas montañosas con ondas fuertes puede afectar toda la troposfera (Nappo, 2002).

Si el aire es seco no hay nubes acompañando a la onda de montaña que permitan su visualización; pero en caso de que haya suficiente humedad, se forman nubes muy típicas que pueden ser estratocúmulo (Sc) y Altocúmulos lenticulares (Ac). Las Nubes a Barlovento, son del tipo estratocúmulo 
(Sc), producidas por la ascendencia del aire húmedo y caliente forzado por la montaña, que luego se enfría y se condensa a cierto nivel, Inmediatamente a sotavento hay descendencias que obligan a la nube a pegarse a la cresta, descendiendo por la ladera escondiendo el nivel de la montaña. El paisaje desde el valle es el de una "muralla nubosa" (muralla de Föehn) o nubes tipo penacho. Corriente abajo, las nuevas ascendencias y descendencias ocasionan la nube rotor, que se forma en la cresta de la onda próxima al nivel del tope de la montaña, subiendo y extendiéndose hacia arriba miles de metros. Esta nube rotor es muy turbulenta. Los Altocúmulos lenticulares (Ac), son las más comunes y constituyen el frente de la onda, a alturas que oscilan entre los 4 y los $9 \mathrm{~km}$. Se forman en las crestas de las ondas, deshaciéndose por calentamiento en las descendencias que forman los vientos.

En las últimas décadas (Alexander y Teitelbaum, 2007; Campetella y Vera, 2002; Fritts y Alexander, 2003), se ha prestado gran atención a las posibles fuentes de ondas de gravedad interna existentes en las atmósferas baja y media. Se sabe que dichas fuentes tienen una notable influencia, tanto en la situación meteorológica local, como en el transporte de cantidad de movimiento y de energía entre distintas alturas, jugando un papel crucial en la circulación general de la tropósfera y la estratosfera (Allen y Vincent, 1995.) Con frecuencia, las ondas de montaña representan un grave peligro para las operaciones aéreas (Linés, 1982) debido a la turbulencia entre fuerte y extrema que pueden producir, a veces sin indicación visual alguna. De allí la importancia de describir las zonas típicas de ocurrencia para revenir al tráfico aéreo.

La longitud de las ondas de montaña puede cambiar hasta un 30\% durante un periodo de doce horas produciéndose ocasionalmente efectos de resonancia. En algunas ocasiones, puede suceder que corriente abajo exista otro obstáculo, si la onda es de tal naturaleza que se ajusta al perfil de la segunda montaña, entra en resonancia y la perturbación aumenta de amplitud, haciéndose, por tanto más intensa corriente abajo más allá del segundo obstáculo.

Las ondas de montaña se forman arriba y a sotavento de las barreras topográficas cuando bajo condiciones de estratificación estable soplan vientos fuertes con un considerable componente vectorial perpendicular a la barrera, lo que fuerza el aire a fluir con una forma sinusoidal, respecto a sus niveles de equilibrio debidas a la flotabilidad. Las parcelas del fluido atmosféricos describirán un movimiento oscilatorio cuya frecuencia limite es la frecuencia de Brunt-Väisälä (Nappo, 2002 y referencias allí citadas).

Para las oscilaciones verticales reales (z) de la parcela del fluido, la Frecuencia de Brunt-Väisälä $(\mathrm{N})$ viene expresadas en términos de la temperatura potencial como (Nappo, 2002):

$$
N=\sqrt{\frac{g}{\theta} \frac{\partial \theta}{\partial Z}}
$$

Donde $g$ es la aceleración de gravedad local y la temperatura potencial $(\theta)$

La Frecuencia de Brunt-Väisälä representa la máxima frecuencia de oscilación vertical de propagación de las ondas de gravedad internas y expresa la estabilidad de una parcela de fluido atmosférico cuando es forzado por el viento a moverse por encima de los accidentes orográficos. La ocurrencia de la onda solo es posible cuando la atmosfera es establemente estratificada, vale decir cuando la frecuencia es real, esto es si $\partial \theta \partial Z>0$. En una atmósfera isotérmica donde $\partial \mathrm{T} / \partial \mathrm{z}=0, \mathrm{~N} \approx 0,02 \mathrm{~s}-1$, y el período de flotabilidad es de aproximadamente 5min. Holton (1992) reporta $\mathrm{N} \approx 0,012 \mathrm{~s}-1$ para las condiciones medias de la troposfera, de modo que el período de flotabilidad es de aproximadamente 8 minutos. En la Capa Limite Planetaria nocturna, $\mathrm{N} \approx 0,2 \mathrm{~s}-1, \mathrm{y}$ los períodos típicos de flotabilidad son del orden de un minuto.

Una forma de caracterizar el movimiento de una parcela de aire ante la cizalladura del viento y la orografía es por medio del parámetro adimensional denominado Número de Froude, que expresa la razón entre la energía cinética y la energía potencial definido como:

$$
\boldsymbol{F r}=\frac{\boldsymbol{V}}{\boldsymbol{N} \boldsymbol{h}_{\max }}
$$

Donde v es la velocidad del viento, hmax es la altura de la barrera y N es la frecuencia de Brunt-Väisäla (Balnes, 1987).

Si el número de Froude equivale o excede ligeramente 1, existe la probabilidad de que se produzcan ondas de montaña, Si en cambio es mucho menor que 1, el flujo de aire no es lo suficientemente fuerte para pasar al otro lado de la cima de la montaña y queda bloqueado: En el caso de valores mucho mayor que 1, el flujo de aire sube por la ladera de la montaña y baja por el otro lado, sin oscilaciones de importancia (Nappo, 2002; Balnes, 1987, Gossard y Hooke, 1975).

El objetivo del presente trabajo es caracterizar las ondas de montaña que ocurren en la capa límite planetaria o troposfera baja en la Región Andina Venezolana correspondiente al período seco y lluvioso entre los años 2006 y 2013. Para ello se discute la metodología en la sección 2, los resultados y su discusión en la sección 3 y, en la última sección se resumen las conclusiones.

\section{2. ÁREA DE ESTUDIO Y METODOLOGÍA.}

En este trabajo se estudian por vez primera las ondas de montaña en la región norte de los Andes de Venezuela, si bien las Ondas de montaña ya han sido estudiadas en la parte sur de Los Andes (de la Torre et al, 2011, Teitelbaum et al, 2008) excluyendo la región andina de Venezuela y Colombia. 
La Cordillera Andina Venezolana comprende los Estados Táchira, Mérida y Trujillo. Esta cadena montañosa se orienta del suroeste (SW) al noreste (NE) con más de $300 \mathrm{Km}$ de extensión y una variedad en cuanto a su elevación que llegan hasta 4981 m.s.n.m. (Pico Bolívar, Edo. Mérida). (Figura 1)

La cadena montañosa del Estado Táchira es de aproximadamente $80 \mathrm{~km}$ de extensión y sus principales elevaciones son el Pico El Pulpito 3942 (m.s.n.m.), Pico la Tigra 3634, Pico El Cobre 3630 m.s.n.m. y Paramo Rosario 3612 m.s.n.m., Los Andes del Estado Mérida presentan una elevación promedio de $4000 \mathrm{msnm}$ a lo largo de su ciento cincuenta kilómetros de zona montañosa que comprenden el páramo de Piedras Blancas (4.737 m.s.n.m), el páramo de Mucuchíes (4.077 m.s.n.m) y el paso El Águila (4.077 m.s.n.m). El Estado Trujillo, tiene entre sus elevaciones principales Tetas de Niquitao 4006 msnm y el paramo de Cende 3625 m.s.n.m, y además se presenta una condición especial, la alineación de dos montañas Boconó y San Lázaro con elevaciones máximas de 3000 y 3600 m.s.n.m. que favorecen el efecto de resonancia de las ondas de montaña.

Se analiza cinco mil setecientas (5700) imágenes de satélite del espectro visible de 1 y 4 km de resolución proveniente del satélite GOES 12,13 de la National Oceanic and Atmospheric Administration (NOAA) y National Aeronautics and Space Administration (NASA) tomadas de los archivos del Servicio de Meteorología de la Aviación Militar Bolivariana (SERMETAVIA) comprendido entre los años 2006, 2007, 2008, 2011, 2012 y mes de enero de 2013, con el objetivo de buscar evidencias de ondas de montañas con manifestaciones de nubes lenticulares sobre sotavento de la cordillera andina , considerando el flujo del viento perpendicular a la cadena montañosa de componente este y sureste.

Localizados los días con presencia de ondas de gravedad (montaña), se procede a analizar, las condiciones dinámicas y termodinámicas de la capa límite planetaria de una muestra del $53 \%$ de todos los casos identificados correspondiente tanto al período seco como el período lluvioso de la Región Andina. La selección de la muestra obedeció a los casos donde se aprecio mejor las características de las nubes lenticulares sobre la cordillera andina a través de las imágenes de satélite exceptuando el período seco donde se analizaron los cuatro casos encontrados. Empleando los resultados del radio sondeo de la estación meteorológica de la Aviación Militar Bolivariana ubicada en San Antonio del Táchira en las coordenadas $7^{\circ} 50^{\prime}$ $17,42^{\prime \prime} \mathrm{N}, 72^{\circ} 26^{\prime} 24,3^{\prime \prime} \mathrm{W}$ se estimó la frecuencia de BruntVäisälä, dada en la Ecuación 1, para obtener los valores típicos del número de Froude Ecuación 2.

De igual manera, los perfiles verticales de viento fueron tomados del radio sondeo de la estación de San Antonio del Táchira y de los niveles de presión del Reanálisis del National Center for Environmental Prediction (NCEP- NOAA) en función de la altura troposférica baja de $765 \mathrm{msnm}$ a $4006 \mathrm{msnm}$ (www.esrl.noaa.gov/psd/data/composites/day), puesto que las manifestaciones de ondas de montañas correspondió al Estado Táchira y en especial el estado Trujillo donde se presenta su máxima elevación Tetas de Niquitao ( 4006 msnm). Se utiliza el programa RAOB 5,6 de radiosonda para analizar los diagramas termodinámicos bajo el formato de Tefigrama lo cual permite determinar los perfiles verticales de temperatura, temperatura potencial, viento (velocidad y dirección), humedad y estabilidad atmosférica. Se procesan las imágenes de satélite y se realiza un estudio de las características topográficas de la Cordillera de los Andes a través del programa Google Earth.

\section{RESULTADOS Y DISCUSIÓN}

El análisis de las imágenes del archivo del Servicio de Meteorología de la Aviación Militar Bolivariana, proveniente del satélite GOES 12,13, evidenciaron la presencia de al menos 15 eventos de Ondas de Montaña asociadas a nubes lenticulares (Tabla 1) en el periodo 2006-2013 para la Región Andina Venezolana en los Estados Trujillo y Táchira. La Figura 2

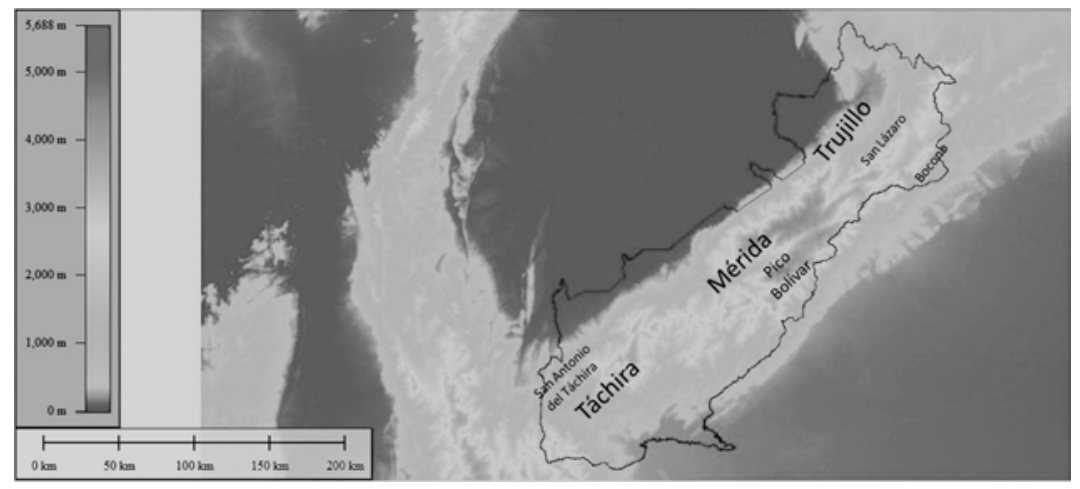

Figura 1: Topografía de la Cordillera Andina Venezolana.

Fuente: Dirección de Geografía y Cartografía de la Fuerza Armada Nacional Bolivariana. 
Tabla 1: Ondas de montaña en presencia de nubes lenticulares.

\begin{tabular}{|c|c|c|c|}
\hline Fecha & Hora(UTC) & Trujillo & Táchira \\
\hline $05 / 07 / 06$ & $15: 45$ & $\mathrm{X}$ & $\mathrm{X}$ \\
\hline $07 / 07 / 11$ & $17: 00$ & & $\mathrm{X}$ \\
\hline $10 / 07 / 11$ & $12: 00$ & $\mathrm{X}$ & \\
\hline $21 / 07 / 11$ & $15: 48$ & $\mathrm{X}$ & $\mathrm{X}$ \\
\hline $07 / 03 / 12$ & $11: 39$ & $\mathrm{X}$ & \\
\hline $08 / 03 / 12$ & $11: 09$ & $\mathrm{X}$ & \\
\hline $06 / 06 / 12$ & $14: 00$ & $\mathrm{X}$ & $\mathrm{X}$ \\
\hline $06 / 07 / 12$ & $15: 00$ & $\mathrm{X}$ & \\
\hline $25 / 07 / 12$ & $11: 00$ & $\mathrm{X}$ & $\mathrm{X}$ \\
\hline $26 / 07 / 12$ & $11: 30$ & $\mathrm{X}$ & $\mathrm{X}$ \\
\hline $29 / 07 / 12$ & $11: 00$ & $\mathrm{X}$ & \\
\hline $28 / 08 / 12$ & $11: 00$ & $\mathrm{X}$ & \\
\hline $13 / 09 / 12$ & $15: 39$ & $\mathrm{X}$ & $\mathrm{X}$ \\
\hline $25 / 11 / 12$ & $12: 30$ & $\mathrm{X}$ & \\
\hline $02 / 01 / 13$ & $11: 45$ & $\mathrm{X}$ & $\mathrm{X}$ \\
\hline
\end{tabular}

muestra imágenes típicas de nubes lenticulares sobre la región Trujillana de Niquitao, tomadas por amateurs en Septiembre del 2012.

Se puede observar (Tabla 1) que todas las manifestaciones de ondas de montaña asociadas a nubes lenticulares (tomadas a través de imágenes de satélite) en la Cordillera Andina ocurrieron en horas de la mañana y eventualmente al mediodía 11:00 -17:00 UTC (06:30-12:30 HLV), su mayor frecuencia corresponde al Estado Trujillo (93\%) y un (53\%) simultáneamente (Trujillo y Táchira).

Los resultados se presentan en forma general, discutiendo su sinóptica en la sección 3.1; y luego se especifican y discuten varios casos conspicuos recientes de Ondas de Montaña en la Región Andina Venezolana en la sección 3.2.

\subsection{Sinóptica General}

En Venezuela se distinguen dos períodos climáticos bien definidos, Verano o estación seca de Noviembre hasta Abril e Invierno o estación lluviosa de Mayo a Octubre
(Goldbrunner, 1984). La temporada lluviosa comienza a finales de Marzo para el estado Trujillo, en la primera quincena de Abril hacia la porción sur del estado Táchira, pero hacia el norte y la localidad de Mérida en la segunda quincena de Abril (Goldbrunner, 1984). En general, durante el trimestre junio-agosto se presenta una disminución de las precipitaciones sobre gran parte de la Cordillera Andina Venezolana. Como muestra en la Figura 3 las ondas de montaña pueden ocurrir en cualquier época del año incluido el periodo seco como en el período lluvioso en la Cordillera Andina Venezolana y su máxima ocurrencia se presenta en el mes de julio.

En la Figura 4 (sup) se representa la climatología de la velocidad del viento (1981-2010) con dirección perpendicular a la Cordillera Andina (Trujillo), a partir del reanálisis de la NOAA. Se evidencia que los meses de junio a agosto; y en especial el mes de julio; se establece climatológicamente sobre la cordillera andina venezolana un flujo de viento perpendicular de máxima intensidad entre $1500 \mathrm{~m}$ a $3000 \mathrm{~m}$. Este máximo de viento en el mes de julio concurre con el máximo de las ondas de montaña encontradas en los años analizados, De igual modo, la alta frecuencia de ondas de montañas sobre la parte norte de la Cordillera Andina (Trujillo), en comparación con la parte sur (Táchira) se podría explicar por dos motivos: en primer lugar, en promedio la velocidad en la parte norte de la cordillera es mayor que en la parte sur (Figura 4 inf), es decir, la energía cinética sobre Trujillo es mucho mayor. En segundo lugar, con el efecto de resonancia debido a la alineación orográfica de las cadenas montañosas de Boconó y San Lázaro en el Estado Trujillo,(Figura 1) con aproximadamente $30 \mathrm{~km}$ de extensión, una separación media entre las montañas de 15 km y una elevación máxima de 3000 m.n.s.m. (Boconó) y 3600 m.s.n.m. (San Lázaro) favorece la sinóptica necesaria para la génesis de ondas de montañas sobre la cordillera andina venezolana.
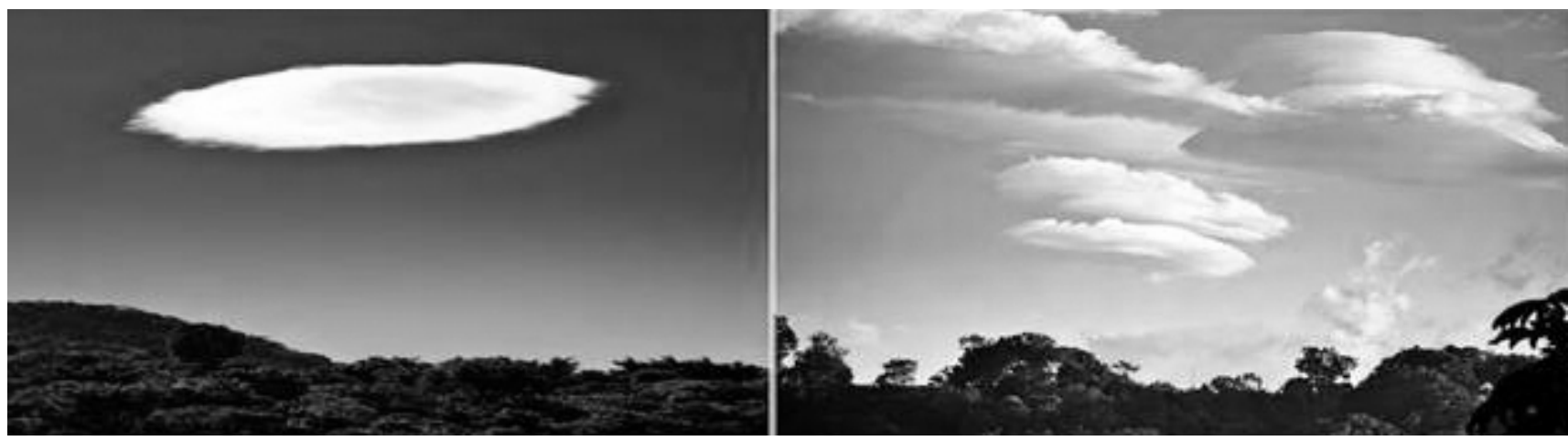

Figura 2: Ejemplos de nubes lenticulares sobre Los Andes Trujillanos. Coordenadas geográficas: 9.205492 N 27.366 W (Izq) y 9.212587 N 26.4118 W (der) .Julio 2012. 


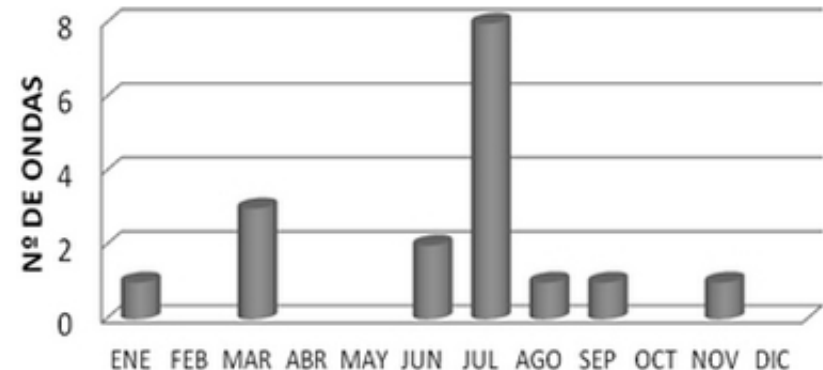

Figura 3: Frecuencia de ondas de montañas en la cordillera andina venezolana. Años 2006 ,2007, 2008, 2012 y 2013.

\subsection{Ejemplo Específicos (Período Seco)}

Los casos analizados en este apartado corresponden a los cuatro casos encontrados durante el período de estudio específicamente en las fechas siguientes; 2013/01/02, 2012/03/07, 2012/03/08 y 2012/11/25, los cuales, en el primer evento se manifiesta ondas de montaña en la parte sur (Estado Táchira) de la Cordillera Andina y en los tres siguientes presenta ondas de montaña simultáneamente en la parte norte y sur (Estado Táchira) de la Cordillera Andina Venezolana.

Caso 2013/01/02. Se observa en la Figura 5a, el perfil vertical del viento tomado del radiosondeo de la estación de San
Antonio del Táchira del 2013/01/02, una inversión de cizalladura del viento (disminución de la velocidad del viento) hasta la cima de la cordillera, con velocidades mayores a $10 \mathrm{~m} / \mathrm{s}$. Entre los 5000 y $7000 \mathrm{~m}$ se aprecia una fuerte cizalladura vertical hacia adelante (aumento del viento con la altura), lo que da origen a vórtices y una atmosfera turbulenta. Esta cizalladura impide la propagación vertical de la onda. En el caso del Radiosondeo del 2013/01/02 en la estación meteorológica de San Antonio del Táchira (Figura 5b) se observa una inversión de temperatura desde los $3436 \mathrm{~m}$ hasta $3774 \mathrm{~m}$. La atmosfera se hace seca y muy estable por encima de dicha inversión. El viento predominante hasta los $8200 \mathrm{~m}$ es de componente E y SE, perpendicular a la cordillera andina. La disponibilidad de humedad por debajo de la inversión de temperatura es significativa ( $>70 \%)$. Con datos aportados del radiosondeo (Figura 5c) de la estación de San Antonio del Táchira se calcula la variación altitudinal del número de Froude que evidencia que a partir de 3500 $\mathrm{m}$, los valores son ligeramente mayor a 1 , favorables para la generación de ondas de gravedad. De acuerdo a la sinopsis de la imagen del satélite del GOES 13 (NASA) del espectro visible de un kilometro de resolución se puede observar (Figura 5d) la presencia de nubes lenticulares sobre la cordillera del Estado Táchira (débiles) y en especial sobre el estado Trujillo (Fuerte), asociadas a ondas de montaña. Estas nubes se encuentran
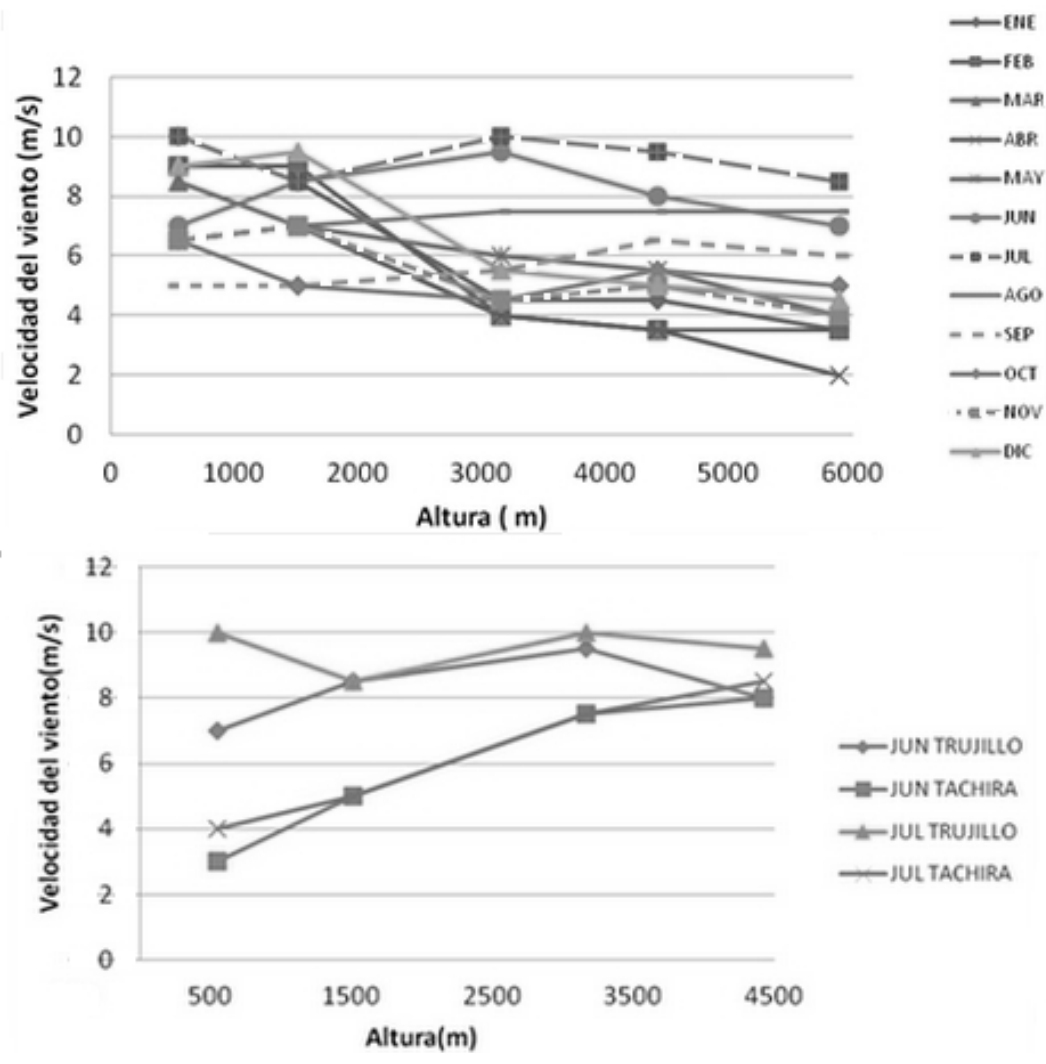

Figura 4: Climatología de la velocidad del vient0, años 1981-2010.(sup) del Estado Trujillo (inf) Comparación entre los Andes de Trujillo y Táchira. 
a)

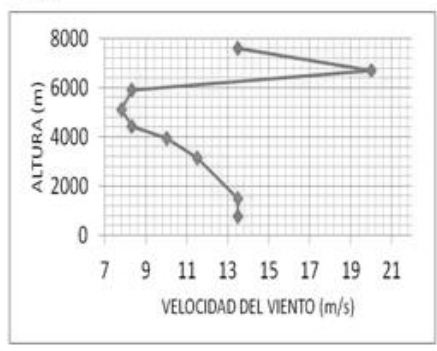

c)

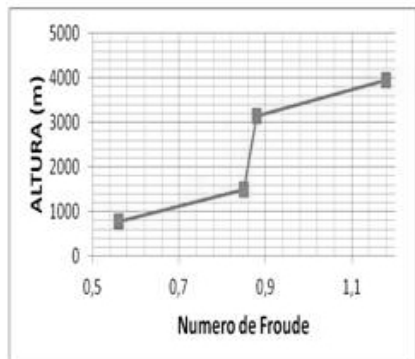

b)

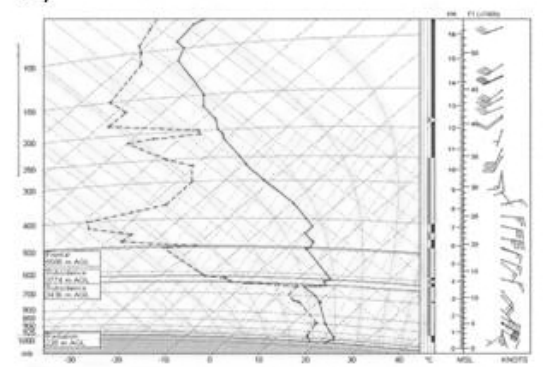

d)

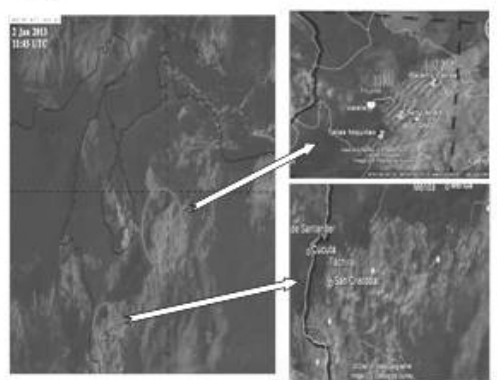

Figura 5: Caso 2013/01/02. Condiciones dinámicas y termodinámicas del 2013/01/02 bajo la presencia de ondas de montañas sobre los Estados Trujillo y Táchira.

a)

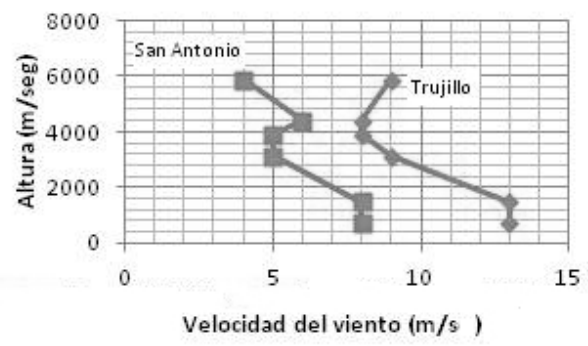

b)

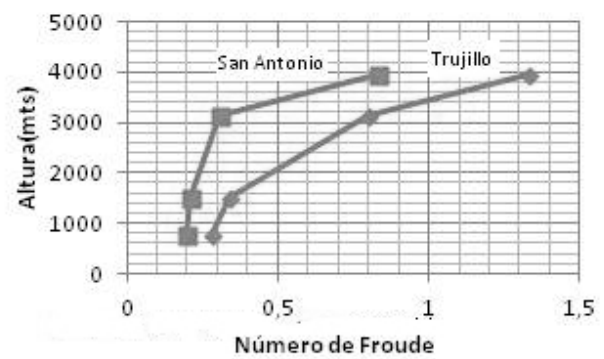

c)

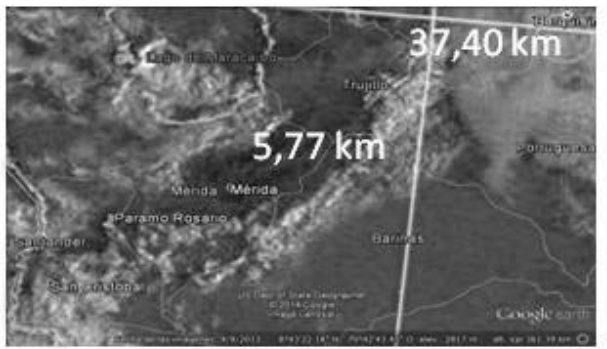

d)
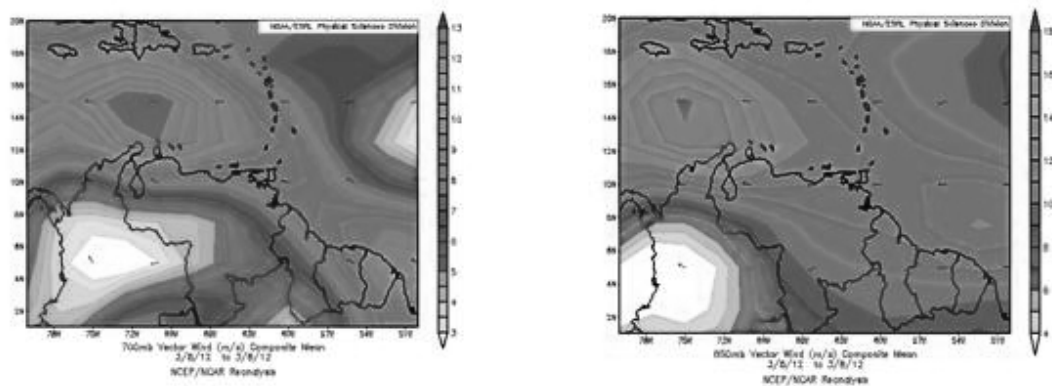

Figura 6: Caso 2012/03/07. Evolución de la dinámica atmosférica del 2012/03/07 en presencia de ondas de montañas sobre el Estado Trujillo. 
alineadas en siete filas a sotavento a partir de la montaña de Bocono con una longitud de onda promedio de 4,42 km y una propagación horizontal de $35 \mathrm{~km}$.

Caso 2012/03/07. Los resultados del perfil vertical del viento (Figura 6a) tomados del reanálisis de la NOAA constata un dominio de inversión de cizalladura tanto en Trujillo como en Táchira hasta una altura de $4000 \mathrm{~m}$. Para el caso de Trujillo, por encima de la cordillera se verifica un aumento de la velocidad del viento. La velocidad del viento es mayor en Trujillo con máximo de $13 \mathrm{~m} / \mathrm{s}$ en contraposición en Táchira cuyo máximo es de $6 \mathrm{~m} / \mathrm{s}$. La Figura 6b, muestra que el numero de Froude (Fr) sobre San Antonio del Táchira hasta la altura de 4000m presenta valores menores a 1 , en cambio, sobre Trujillo se observa valores superiores a 1 , favorables para la manifestación de ondas de gravedad. También se observa en la imagen del espectro visible de satélite GOES 13 (Figura 6c) el predominio de nubes lenticulares a sotavento del estado Trujillo con una extensión horizontal de $50 \mathrm{~km}$ y una longitud de onda promedio de 6 km. Sobre el estado Táchira no se aprecia evidencia clara de nubes lenticulares. Finalmente, la Figura 6d correspondiente

a)

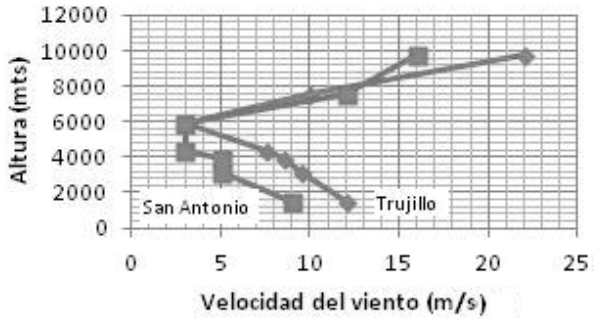

b)

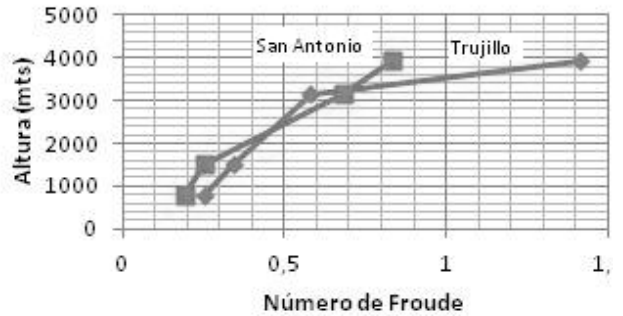

al reanálisis (NCEP-NOAA) muestra la existencia de viento perpendicular con dirección este sobre la cordillera andina a diferentes alturas.

Caso 2012/03/08. Se ilustra en la Figura 7a, el perfil vertical del viento en base al reanálisis del NCEP/NOAA del día 2012/03/08 la presencia de inversión de cizalladura en la cordillera de los estados Trujillo y Táchira hasta los $6000 \mathrm{~m}$, por encima de este nivel se aprecia cizalladura vertical hacia adelante. Se repite, como en casos anteriores, la velocidad del viento del estado Trujillo supera en todos los niveles al estado Táchira. De acuerdo, a la Figura 7b, el número de Froude correspondiente a San Antonio del Táchira no supera el umbral para desencadenar ondas de gravedad, en cambio, sobre el estado Trujillo se reporta Fr $>1$ favorable para la génesis de ondas de gravedad. De igual modo, se verifica en la Figura 7c, correspondiente a la imagen del espectro visible de satélite GOES 13, la presencia de nubes lenticulares a sotavento de la cordillera andina específicamente sobre el estado Trujillo con una extensión aproximada de 37,4km y una longitud de onda promedio de 5,8 .km. Sobre la cordillera del estado Táchira no

c)

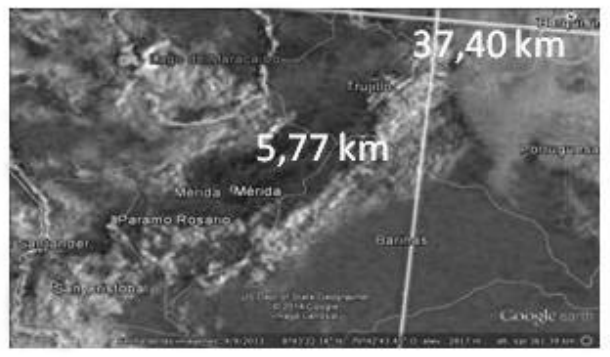

d)
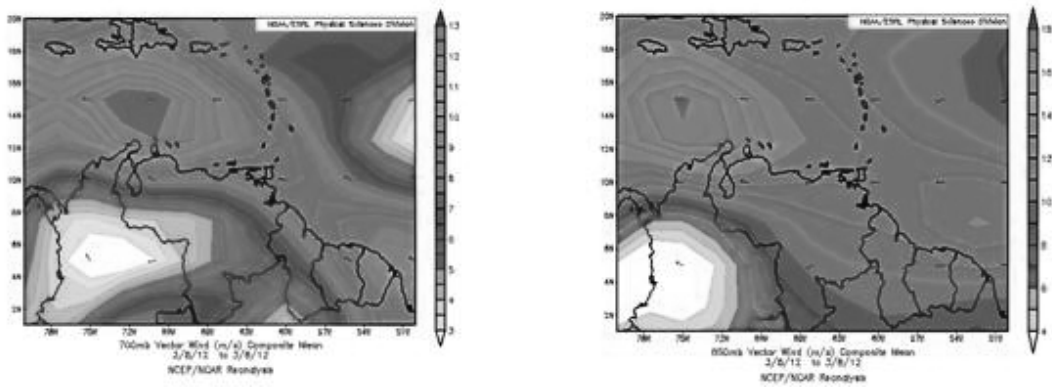

Figura 7: Caso 2012/03/08. Dinámica atmosférica del 2012/03/07 en presencia de ondas de montañas sobre el Estado Trujillo con datos del reanálisis del NCEP/NOAA y del satélite GOES 13. 
se observa evidencia clara de nubes lenticulares. Finalmente en la Figura 7d, del reanálisis de la NOAA se constata la dirección de componente este y sureste a lo largo de la cordillera andina.

Caso 2012/11/25. El perfil vertical del viento, con datos del reanálisis, muestra que todos los valores del estado Trujillo son mayores a los de San Antonio del Táchira (Figura 8a). En el caso de Trujillo varían entre 9 y $12 \mathrm{~m} / \mathrm{s}$ y en Táchira entre 5 y $9 \mathrm{~m} / \mathrm{s}$. También sobre el Estado Táchira se verifica predominio de cizalladura vertical hacia adelante hasta los 4500 $\mathrm{m}$ y en Trujillo inversión de cizalladura vertical exceptuando por debajo de $1400 \mathrm{~m}$. Por su parte en la Figura 8b se demuestra que los valores de la variación del número de Froude sobre el estado Táchira están por debajo de 1, en cambio, sobre el estado Trujillo especialmente por encima de $3000 \mathrm{~m}$, se aprecia valores superiores a 1 , favorables para la génesis de ondas de gravedad. En la sinopsis de la imagen de satélite GOES 13, del espectro visible del día 2012/11/25 (Figura 8c) se evidencia la presencia de nubes lenticulares alineadas arriba y a sotavento de la cordillera del estado Trujillo con una extensión horizontal aproximada de $38 \mathrm{~km}$ y una longitud de onda promedio de 5,5 km. No se aprecia nubes lenticulares sobre el estado Táchira. Por último, se observa la prevalencia de vientos de componente este y sureste en la capa limite planetaria de la región andina venezolana (Figura 8d).

\subsection{Ejemplo Específicos (Período Lluvioso)}

Los casos de estudios corresponden aproximadamente al $40 \%$ de los casos encontrados durante el período lluvioso específicamente en las fechas siguientes; 2011/07/21, 2006/07/06, 2012/06/06 y 2012/07/06, los cuales, los tres primeros eventos manifiestan ondas de montaña no solamente en la porción norte (Estado Trujillo) sino también en la porción sur (Estado Táchira) de la Cordillera Andina Venezolana y el último caso de estudio refleja ondas de montaña solamente en el Estado Trujillo.

Caso 2011/07/21. La Figura 9a ilustra el perfil del viento con datos del radiosondeo de la estación de San Antonio del Táchira, una atmosfera esencialmente con cizalladura vertical (propicia para el origen de turbulencia). Desde superficie

b)

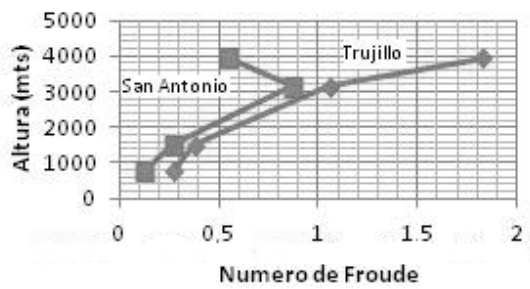

Numero de Froude
Velocidad del viento $(\mathrm{m} / \mathrm{s})$

a)

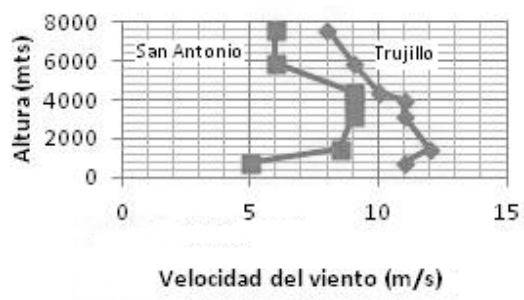

c)

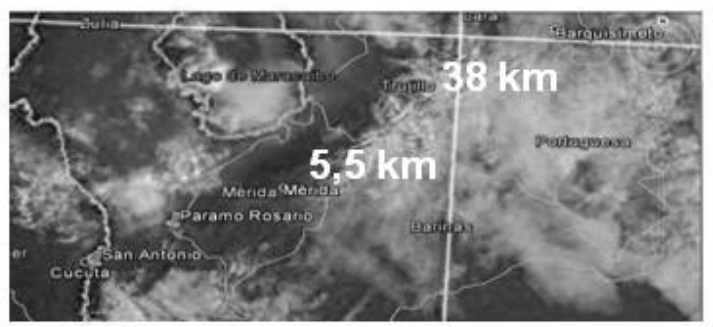

d)
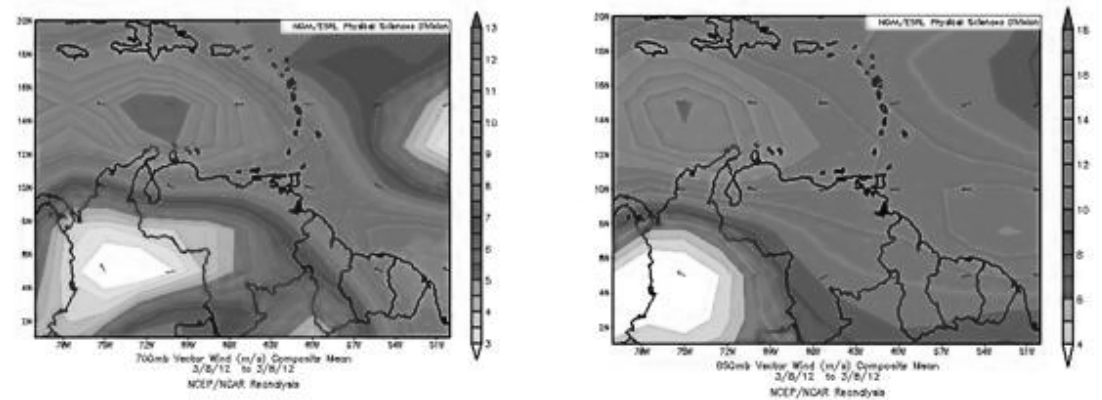

Figura 8: Caso 2012/11/25. Dinámica atmosférica del 2012/11/25 en presencia de ondas de montañas sobre el Estado Trujillo con datos del reanálisis NCEP/NOAA y imagen del satélite GOES 13. 
a)

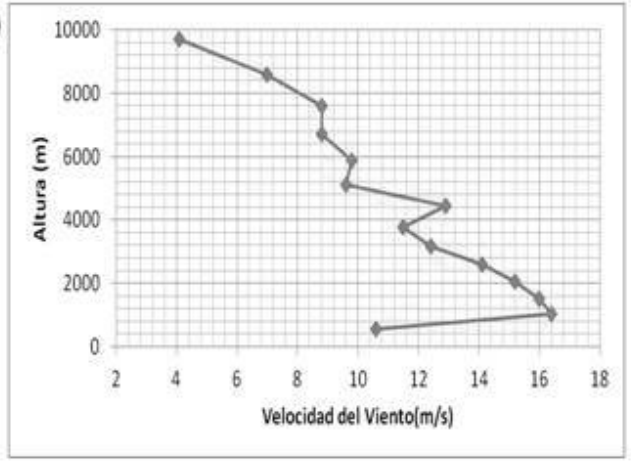

c)

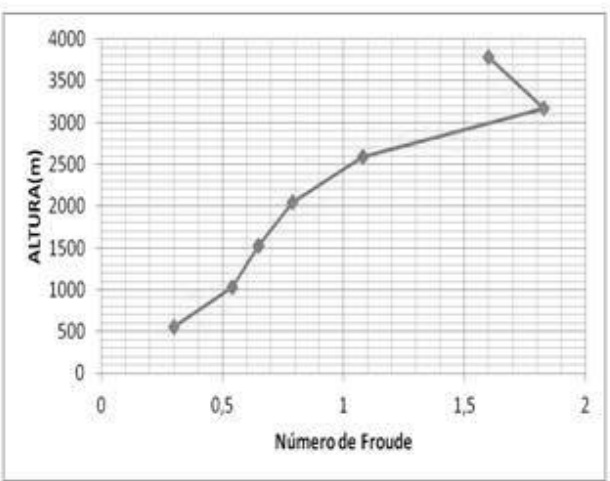

b)

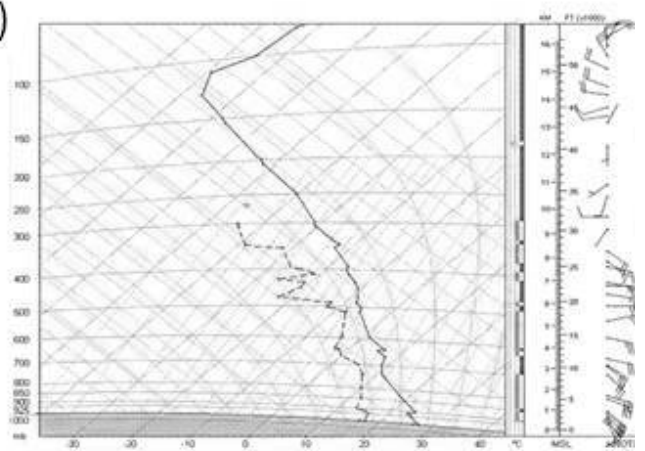

d)

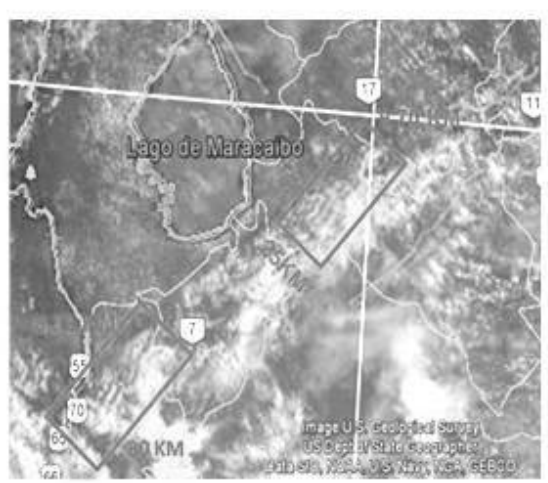

Figura 9: Caso 2011/07/21. Condiciones dinámicas y termodinámicas del 2011/07/21 en presencia de ondas de montañas sobre los Estados Trujillo y Táchira en base a datos del radiosondeo de San Antonio del Táchira y del satélite del GOES 13.

hasta los $4000 \mathrm{~m}$ el flujo del viento es mayor a $10 \mathrm{~m} / \mathrm{s}$. Se aprecia predominio de inversión de cizalladura vertical. Según el análisis del diagrama termodinámico del 2011/07/21, de San Antonio del Táchira (Figura 9b) presenta una atmosfera generalmente estable con dos pequeñas inversiones de temperatura una aproximadamente en los $900 \mathrm{hPa}(612 \mathrm{~m})$ y otra hacia los 650 hPa (3527 m).Por encima de los $4000 \mathrm{~m}$ disminuye la estabilidad y se observa una capa neutra entre 4000 y $5500 \mathrm{~m}$. La atmosfera carece de humedad relativa significativa exceptuando los niveles de $700 \mathrm{hPa}$ ( $2900 \mathrm{~m}$ ) con $75 \%$ y 500 hPa (5500 m) con 80\%.Asimismo, se aprecia viento de componente E y SE desde superficie hasta los $350 \mathrm{hPa}$ (8400 m). Con los datos del radiosondeo, se elaboró el grafico del número de Froude, Figura 9c, que presenta Fr $>1$ por encima de $2500 \mathrm{~m}$, favorables para desencadenar ondas de gravedad. De acuerdo con la imagen visible del GOES 13 (NOAA) se puede observar la presencia de nubes lenticulares sobre la cordillera de los Estados Táchira y Trujillo, asociadas a ondas de montaña (Figura 9d).Estas nubes se encuentran alineadas en cuatro bandas. Para Táchira se observa una longitud de onda promedio de 8,92 km y una propagación horizontal de $30 \mathrm{~km}$ y en Trujillo la longitud de onda promedio es de 9,70 km y una propagación horizontal aproximada de $25 \mathrm{~km}$ a sotavento de la Cordillera Andina.
Caso 2006/07/05. El perfil del viento en base al reanálisis del NCEP, correspondiente al Estado Trujillo, (Figura 10a) se aprecia un incremento de la velocidad del viento de $10 \mathrm{~m} / \mathrm{s}$ en la superficie, hasta los $18,5 \mathrm{~m} / \mathrm{s}$ a $3000 \mathrm{~m}$. Por encima de los $3000 \mathrm{~m}$ hasta los $6000 \mathrm{~m}$ se presenta una disminución de la velocidad del viento que alcanza los $13 \mathrm{~m} / \mathrm{s}$. Esta inversión de cizalladura origina vórtices y turbulencia que favorece la propagación vertical de la energía de la onda de gravedad. Con los datos del reanálisis se puede calcular el número de Froude, como se muestra en la Figura 10b. Se aprecia Fr $>1$ por encima de los $3000 \mathrm{~m}$, favorables para el origen de ondas de montañas en ambos estados: Táchira y Trujillo. En relación a la imagen del espectro visible del satélite GOES 12, del 2006/07/05 de un kilometro de resolución a las 15:45 UTC (Figura 10c) se observa sobre la cordillera de Trujillo seis bandas alineadas de nubes lenticulares asociadas a ondas de montaña con una longitud promedio de 9,66 km y una propagación horizontal de aproximadamente $50 \mathrm{~km}$ a sotavento. Para este evento no se tienen los datos del radiosondeo pero se puede observar a través de las imágenes del reanálisis del NCEP/NOAA a diferentes alturas la dirección del viento (Figura 10d) de componente este y sureste perpendicular a la Cordillera Andina Venezolana.

Caso 2012/06/06. En concordancia a la Figura 11a, referido al reanálisis NCEP el perfil vertical del viento sobre 
a)

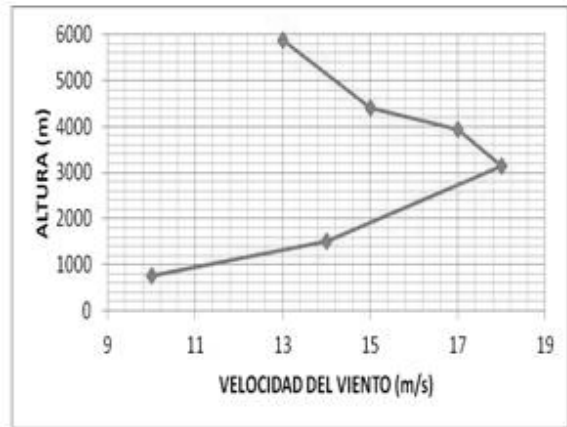

b)

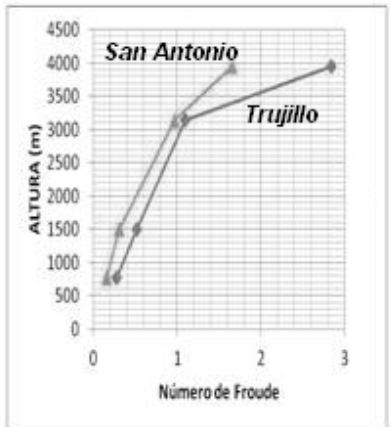

c)

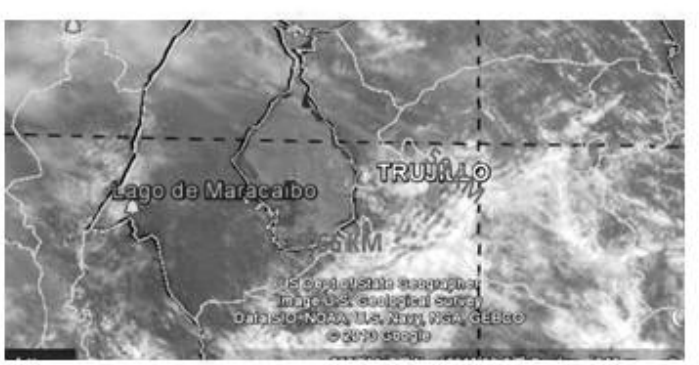

d)
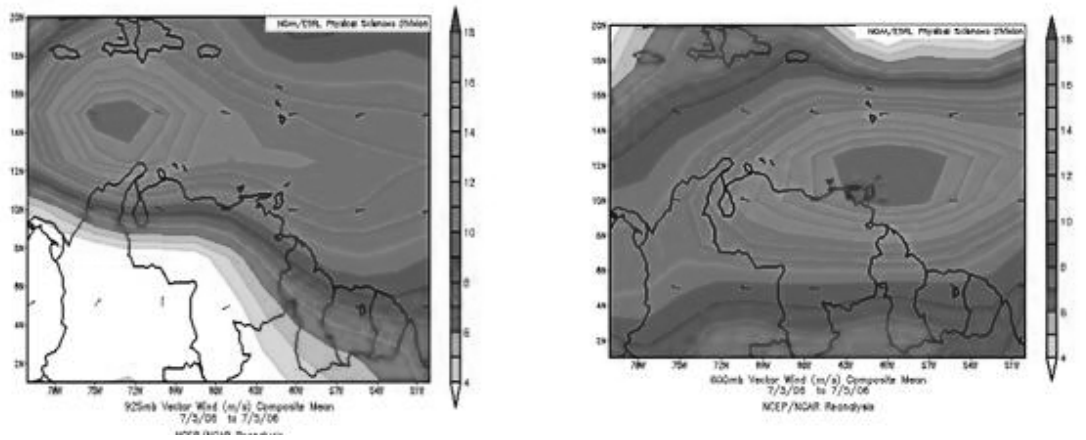

Figura 10: Caso 2006/07/05. Dinámica atmosférica del 2006/07/05 en presencia de ondas de montañas sobre el Estado Trujillo con datos del reanálisis NCEP/NOAA y del satélite GOES 12.

el estado Trujillo se observa con predominio de inversión de cizalladura vertical y en San Antonio del Táchira predomina cizalladura vertical hacia adelante hasta la cima de la montaña y por encima se aprecia inversión de cizalladura vertical. Por otra parte, se ilustra en la Figura 11b, la variación con respecto a la altura del número de Froude con valores superiores a 1, entre los 3000 y $4000 \mathrm{~m}$ tanto para el estado Trujillo como Táchira, favorable para el origen de ondas de gravedad. Con respecto a la sinopsis de la imagen del satélite GOES 12 (Figura 11c) del canal visible se observa presencia de nubes lenticulares a sotavento de la cordillera de Trujillo y Táchira, con una extensión horizontal aproximada de 48,2 km y longitud de onda promedio de $9 \mathrm{~km}$ para el estado Trujillo y una extensión horizontal de $27 \mathrm{~km} \mathrm{y}$ longitud de onda de $8,22 \mathrm{~km}$ para el estado Táchira. Se constata el flujo de viento de componente E y ESE a lo largo de la cordillera andina venezolana (véase Figura 11d).

Caso 2012/07/06. Según la Figura 12a, en referencia al perfil vertical del viento se observa cizalladura vertical hacia adelante tanto en el estado Trujillo como en el estado Táchira, desde superficie hasta $4000 \mathrm{~m}$ que corresponde aproximadamente la cima de la cordillera, por encima de dicha altura prevalece inversión de cizalladura vertical especialmente sobre la cordillera del estado Trujillo. De igual manera, se identifica a lo largo de la cordillera del estado Trujillo velocidades del viento oscilando entre 10 a $16 \mathrm{~m} /$ seg. Se ilustra en la Figura 12b, valores del número de Froude superiores a 1, entre 3000 a 4000 m calculados en referencia al reanálisis correspondientes a los estados Táchira y Trujillo. Por otra parte, en la imagen visible del satélite GOES 12 (Figura 12c) se observa la presencia de nubes lenticulares al norte de la cordillera del estado Mérida y a lo largo de la cordillera del estado Trujillo con una extensión horizontal aproximada de $36 \mathrm{~km}$ y una longitud de onda promedio de $10 \mathrm{~km}$, nótese que de las imágenes de satélite estudiadas representa el único caso donde se observa la presencia de nubes lenticulares sobre el estado Mérida. Por último, se certifica en la Figura 12d, la 
a)

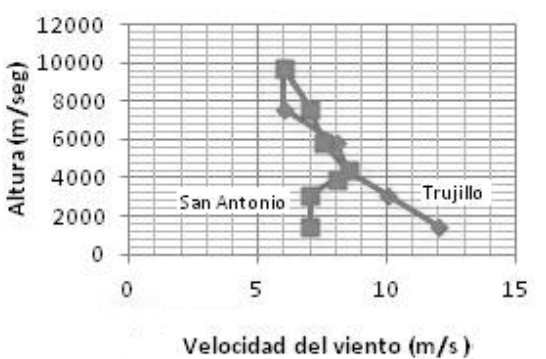

b)

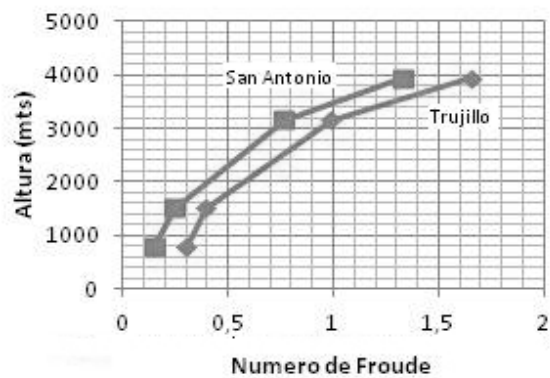

c)

d)
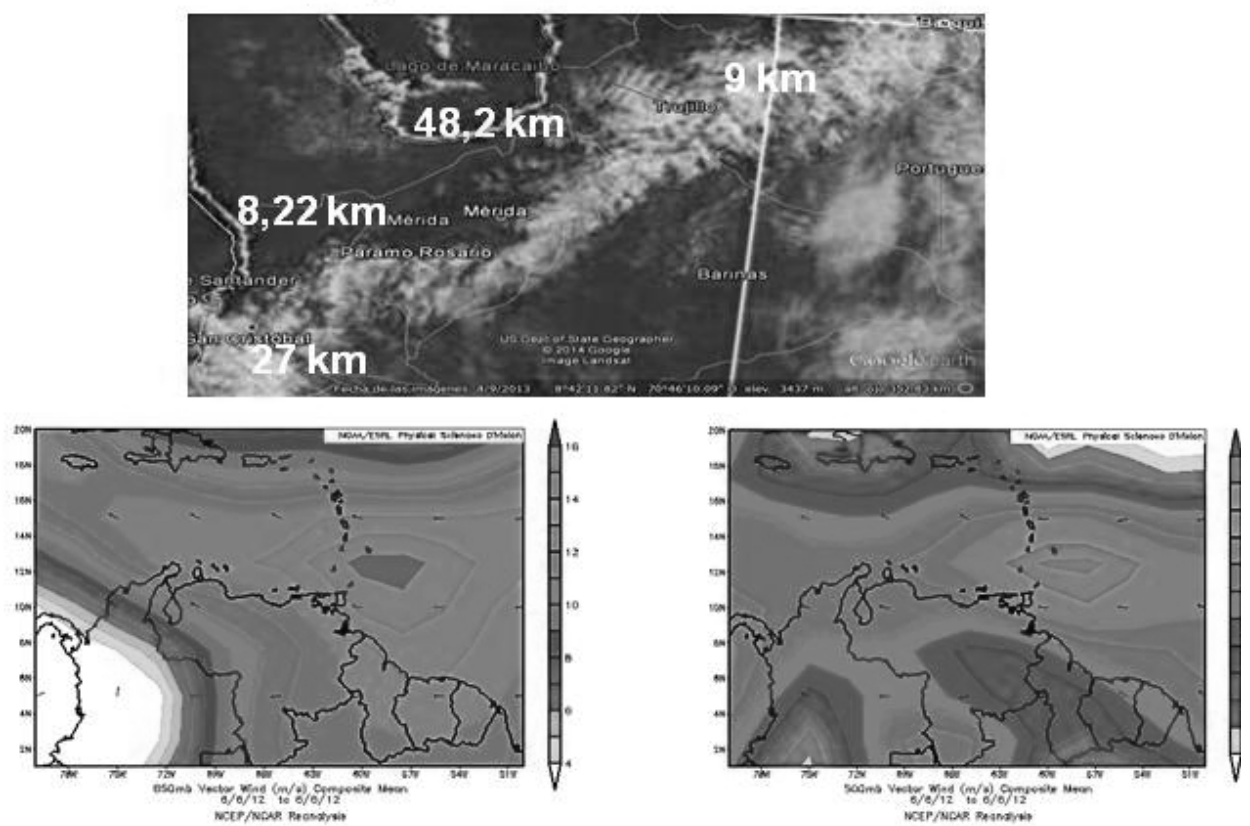

Figura 11: Caso 2012/06/06. Condiciones atmosférica del 2006/07/05 en presencia de ondas de montañas sobre los Estados Táchira y Trujillo con datos del reanálisis NCEP/NOAA y del satélite GOES 12.

Tabla 2: Análisis de Medias de la de ondas de montaña sobre la Cordillera Andina Venezolana

\begin{tabular}{|lccc|cccc|}
\hline \multicolumn{4}{|c|}{ Longitud } & \multicolumn{3}{|c|}{ Propagación horizontal a sotavento } \\
\hline Ondas & $\mathrm{N}$ & Media & grupación & \multicolumn{2}{|c|}{ Ondas } & N & Media Agrupación \\
\hline 2 & 5 & 9,3160 & A & 1 & 4 & 40,100 & A \\
\hline 1 & 4 & 5,4225 & B & 2 & 6 & 36,033 & A \\
\hline
\end{tabular}

influencia de vientos de componente este y sureste en la capa baja de la troposfera.

Finalmente, se realiza un análisis de medias de las longitudes de ondas y de propagación horizontal de las ondas de montaña a sotavento de la cordillera andina en referencia a todos los casos estudiados del período seco y lluvioso a través del método de Tukey con un nivel de confianza de $95 \%$, el cual, permite determinar que las longitudes de ondas del período lluvioso presentan diferencias significativas en relación a las longitudes de ondas del período seco (Tabla 2). En cuanto a la propagación horizontal de las ondas de montaña a sotavento con evidencia de nubes lenticulares no presentan diferencias significativas.

\section{CONCLUSIONES.}

La manifestación de las nubes lenticulares a sotavento de la Cordillera Andina Venezolana es una evidencia física de la 
a)

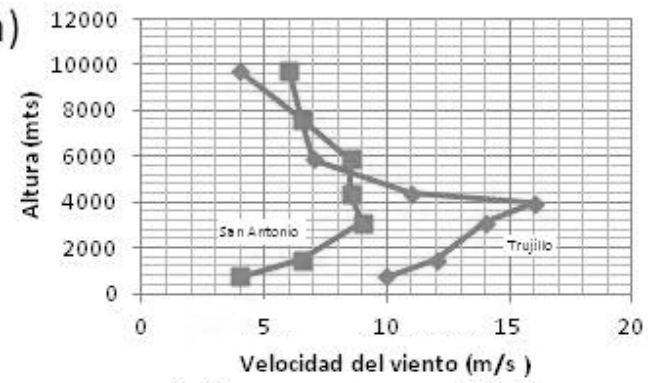

b)

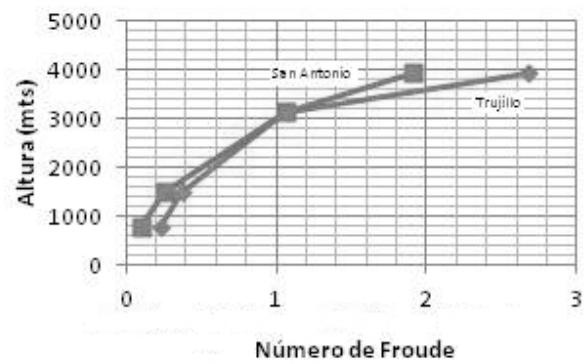

c)

d)
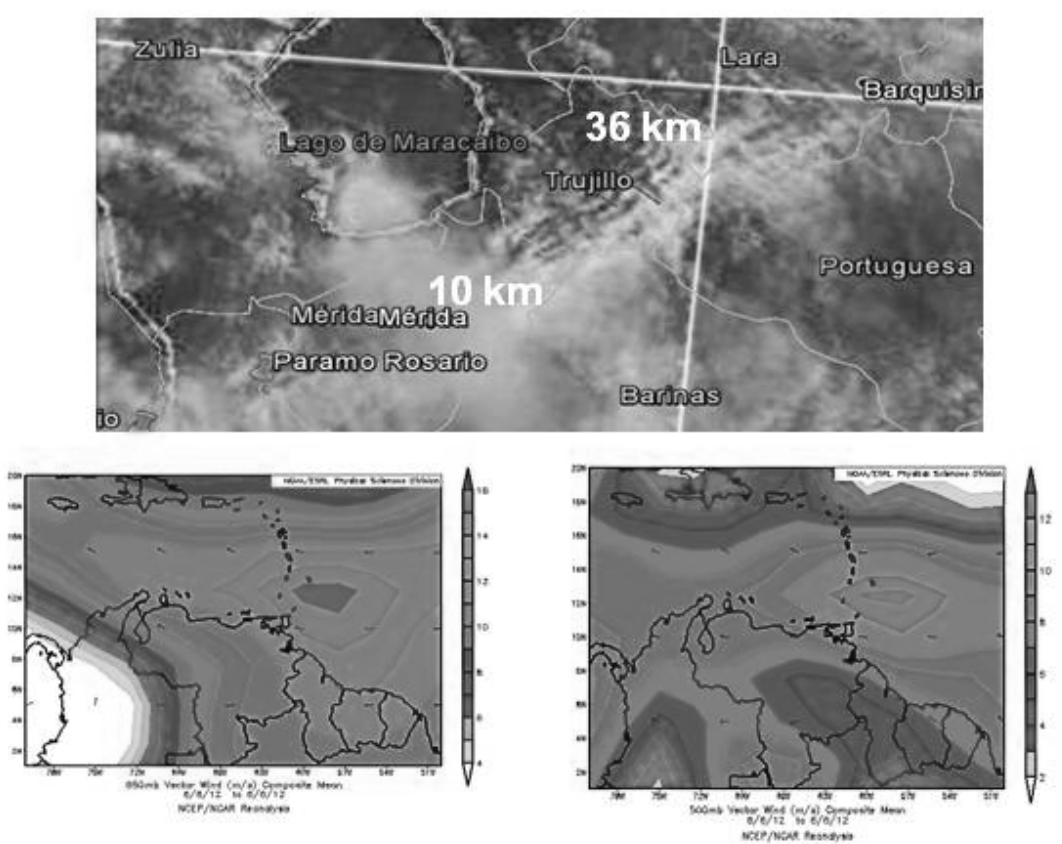

Figura 12: Caso 2012/07/06. Dinámica atmosférica del 2006/07/06 bajo la influencia de ondas de montañas sobre el Estado Trujillo con datos del reanálisis NCEP/NOAA y del satélite GOES 12.

presencia de ondas de montaña. El perfil vertical del viento a lo largo de la Cordillera Andina (Táchira y Trujillo) con dirección E o SE y velocidades mayores de $10 \mathrm{~m} / \mathrm{s}$ y numero de Froude ligeramente superior a 1 son buenos referentes para predecir la ocurrencia de ondas de montaña.

Las ondas de montaña en el período seco se presentan con profunda inversión de temperatura arriba de la cima de la cordillera, mientras que en el periodo húmedo no se presenta dicha inversión, como se evidencia en las Figuras 6 y 12. Las ondas de montaña en el período seco exhiben un predominio de inversión de cizalladura vertical desde superficie hasta la cima de la montaña, y sobre la cordillera una frecuente cizalladura vertical hacia adelante; en cambio, las ondas de montaña del período lluvioso tienen un perfil vertical de viento con cizalladura vertical hacia adelante desde superficie hasta la cima de la montaña y recurrencia de inversión de cizalladura vertical por encima de la montaña. Las longitudes de ondas en el periodo lluvioso son más largas (en promedio
9,3 km) en comparación con el período seco (5,4km), y son significativamente diferentes, debido posiblemente a la mayor intensidad del viento en el período lluvioso especialmente en el mes de julio y mayor estabilidad en la capa baja de la troposfera en el período seco.

La propagación horizontal de las ondas de montaña con manifestación de nubes lenticulares a sotavento del Estado Trujillo (parte norte de la Cordillera Andina) tanto para el período seco como el lluvioso no presentan diferencias significativas y en promedio es de $38 \mathrm{~km}$. La mayor frecuencia de ocurrencia de ondas de montaña asociadas a nubes lenticulares observadas en el estado Trujillo en comparación al estado Táchira, obedece posiblemente al establecimiento climatológico en el estado Trujillo de un viento más intenso en la capa límite planetaria y al efecto de resonancia producido por la alineación de las montañas de Boconó y San Lázaro. La orografía particular de la región andina Venezolana, condiciona los perfiles de velocidad del viento en el periodo seco (diciembre- marzo) y 
lluvioso (abril-noviembre), con números de Froude mayores a la unidad, y en consecuencia la estabilidad es mayor en el periodo seco. Se esperaría un comportamiento similar en otras áreas de la cordillera andina sudamericana; de modo que el seguimiento de patrones de nubes lenticulares podría servir para la predicción de ondas de montaña y su vinculación con las turbulencias orográficas.

\section{AGRADECIMIENTOS.}

Se agradece al Cnel(r) Antonio Magdaleno, Cnel. Julio Cabanerit y May. Luis Vargas del Servicio de Meteorología de la Aviación Militar Bolivariana por los datos de radiosondeo e imágenes de satélites. Se agradece el financiamiento a través del Proyecto Estratégico FONACIT N 2011-000326 "Caracterización de Fenómenos Transitorios en la Troposfera baja: Electrometeoros, Litometeoros, Microtornados y trombas marinas".

\section{REFERENCIAS BIBLIOGRÁFICAS.}

ALEXANDER, M.J.; TEITELBAUM, H. Observation and analysis of a large amplitude mountain wave event over the Antarctic peninsula, Journal of Geophysical Research, v. 112, p. D21103. 2007.

ALLEN, S.J.; VINCENT, R.A. Gravity wave activity in the lower atmosphere: Seasonal and latitudinal variations, Journal of Geophysical Research, v. 100, p. 1327-1350. 1995.
BALNES, P. G. Upstream blocking and airflow over mountain. Annual Review of Fluid Mechanicsm, v. 19, p. 75-97. 1987

CAMPETELLA, C.; VERA, C. The influence of the Andes mountains on the South America low-level flow.

Geophysical Research Letters, v. 29, n. 17, p. 1826. 2002 DE LA TORRE, A. R.; HIERRO, P.; LLAMEDO, A.; ROLLA, P.; ALEXANDER, M. Severe Hail storms near Southern Andes in the Presence of Mountain Waves. Atmospheric Research, v. 101, n.1-2, p. 112-123. 2011.

FRITTS, D.C.; ALEXANDER, M.J. Gravity wave dynamics and effects in the middle atmosphere, Reviews of Geophysics, v. 411, p. 1003. 2003.

GOSSARD, E. E.; HOOKE, W. H. Waves in the Atmosphere, 456 pp. San Diego: Academic Press. 1975.

GOLDBRUnNer, A. Atlas Climatológico. Servicio de Meteorología de la Aviación Militar.Edición .1984.

HOLTON, J. R. An introduction to dynamic meteorology. International geophysics series, San Diego, New York: Academic Press, 1992.

LINÉS E. A. Geographicalia, ISSN 0210-8380, N 13-16, p. 103-116. 1982.

NAPPO, C.J. An Introduction to Atmospheric Gravity Waves, Int. Geophys. Ser., 8., San Diego, California. 2002.

TEITELBAUM, H.; LE TREUT H.; MOUSTAOUI M.; CABRERA, G. C.; IBAÑEZ G. Deep convection east of the Andes Cordillera: A test case analysis of airmas origin. Monthly Weather Review, v. 136, p. 2201- 2209. 2008. 